\title{
Impact of Breast Cancer Resistance Protein Expression on the In Vitro Efficacy of Anticancer Drugs in Pancreatic Cancer Cell Lines $^{\mathbb{S}}$
}

\author{
Ikumi Washio, Takeo Nakanishi, Naoki Ishiguro, Norio Yamamura, and Ikumi Tamai
}

Faculty of Pharmaceutical Sciences, Institute of Medical, Pharmaceutical and Health Sciences, Kanazawa University, Kanazawa, Japan (I.W., T.N., I.T.); and Pharmacokinetics and Non-Clinical Safety Department, Nippon Boehringer Ingelheim Co. Ltd., Kobe, Japan (I.W., N.I., N.Y.)

Received September 5, 2017; accepted December 4, 2017

\section{ABSTRACT}

Breast cancer resistance protein (BCRP) overexpression confers multidrug resistance to cancer cells, and the efficacy of anticancer drugs has been reported to be significantly affected by BCRP in cell lines transfected with $B C R P$ or selected with drugs. It is unclear whether the in vitro efficacy of anticancer drugs is affected by endogenous BCRP, although cancer cell line panels consisting of defined tumor cell lines with endogenous BCRP have been used to screen for anticancer drugs in the pharmaceutical industry. We assessed the impact of BCRP expression on efficacy of anticancer drugs using pancreatic cancer cell lines expressing varying levels of endogenous BCRP. Pancreatic cancer cell lines were selected from the Cancer Cell Line Encyclopedia (CCLE). The EC Fo $_{5}$ of 7-ethyl10-hydroxycamptothecin ( $\mathrm{SN}-38$ ), topotecan, and mitoxantrone decreased in the presence of a BCRP inhibitor in PANC-1 and AsPC1 cells, which exhibit high BCRP expression. However, no significant alterations in $\mathrm{EC}_{50}$ were observed in HPAF-II, SW 1990, and MIA PaCa-2, which show moderate or low BCRP expression. The shift of $E_{50}$ of anticancer drugs with and without a BCRP inhibitor increased with an increase of BCRP mRNA expression levels; however, the shift was obvious only in cells highly expressing BCRP. Thus, the in vitro efficacy of anticancer drugs on cell proliferation may be minimally affected by BCRP in most pancreatic cancer cell lines, considering that $72 \%$ of pancreatic cancer cell lines in CCLE show moderate or low BCRP expression. The effect of BCRP should be carefully evaluated in pancreatic cell lines that highly express BCRP.

\section{Introduction}

Breast cancer resistance protein (BCRP) was discovered as a transporter overexpressed in a multidrug-resistant human breast cancer cell line (Doyle et al., 1998). BCRP has been reported to recognize many anticancer drugs as substrates, such as the cytotoxic drugs capecitabine (Zhang et al., 2015), methotrexate (Volk et al., 2000), 7-ethyl-10-hydroxycamptothecin (SN-38) (Nakatomi et al., 2001), topotecan (Maliepaard et al., 2001), mitoxantrone (Miyake et al., 1999), etoposide (Allen et al., 2003), and teniposide (Lin et al., 2011), and the molecular-targeted drugs dinaciclib (Cihalova et al., 2015), flavopiridol (Nakanishi et al., 2003a), and imatinib (Burger et al., 2004), and pump these drugs out of cells. Thus, BCRP is known to be involved in multidrug resistance in cancer cells (Nakanishi and Ross, 2012). Until now, cell lines that overexpress BCRP, such as BCRP-transfected and drug-selected cells, have been used for in vitro studies to investigate BCRPmediated drug resistance, and it has been reported that the efficacy of anticancer drugs that are substrates for BCRP can be significantly altered by BCRP. For example, MCF-7/AdrVp cells that were selected by the incremental increase of adriamycin in the presence of a P-glycoprotein ( P-gp) inhibitor, verapamil, and MCF-7 cells stably transfected with $B C R P$

This study was supported by Nippon Boehringer Ingelheim Co., Ltd. https://doi.org/10.1124/dmd.117.078402.

S This article has supplemental material available at dmd.aspetjournals.org.
cDNA (MCF-7/BCRP) showed 3900-fold and 32-fold higher $\mathrm{LC}_{50}$ of mitoxantrone, respectively, than that in nontransfected MCF-7 cells (Doyle et al., 1998).

However, it is unclear whether the efficacy of anticancer drugs is affected by endogenous BCRP expression, although in pharmaceutical industries, cancer cell line panels consisting of well defined tumor cell lines without enforced or in vitro-induced BCRP expression produced under artificial conditions have been frequently used to screen for anticancer drugs (Shoemaker, 2006; Niu and Wang, 2015). In drug discovery and development, the current standard procedure often starts with in vitro cell culture-based tests to screen for the efficacy of drugs, but only approximately $10 \%$ of the drugs progress successfully through clinical development (Edmondson et al., 2014). To increase the success rate of clinical trials, accurate and efficient in vitro screening of anticancer drugs is required. However, currently it remains unclear whether clinically promising drug candidates may be excluded during screening using cancer cell line panels, due to the effect of native BCRP.

The Cancer Cell Line Encyclopedia (CCLE) provides public access to mRNA expression data of approximately 1000 cancer cell lines (Barretina et al., 2012). When mRNA expression levels of BCRP were examined in cancer cell lines using the CCLE database, it was found that endogenous $B C R P$ expression varies largely among the cell lines (Fig. 1; Supplemental Table 1). In the present study, we assessed the impact of BCRP expression on the efficacy of anticancer drugs using several

ABBREVIATIONS: BCRP, breast cancer resistance protein; CCLE, Cancer Cell Line Encyclopedia; FBS, fetal bovine serum; FTC, fumitremorgin C; MDCKII, Madin-Darby canine kidney; OD, optical density; PBS, phosphate-buffered saline; PCR, polymerase chain reaction; P-gp, P-glycoprotein; $\mathrm{RR}$, resistance ratio; SI, signal intensity; SN-38, 7-ethyl-10-hydroxycamptothecin. 


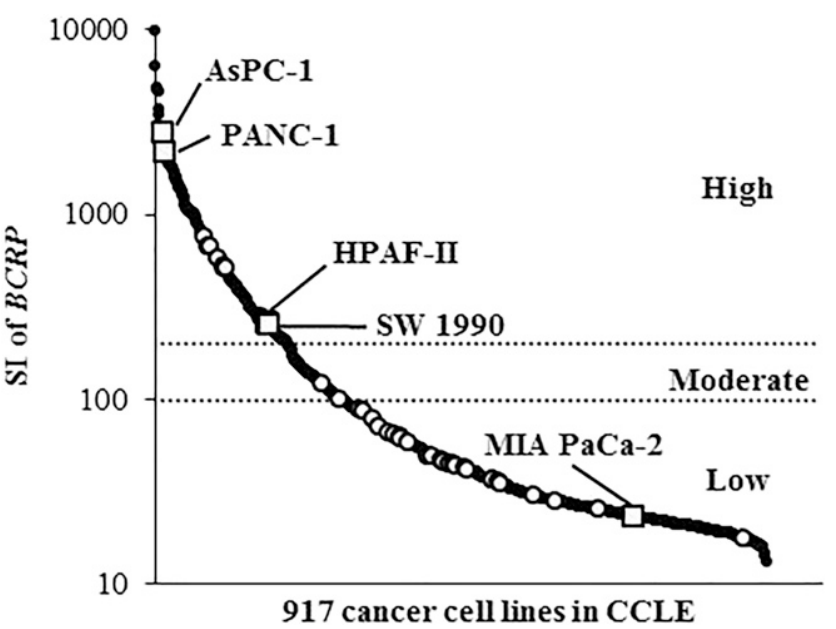

Fig. 1. mRNA expression levels of $B C R P$ represented as SI of microarray analysis in 917 cancer cell lines are arranged in descending order (open square, pancreatic cancer cell lines used in this study; open circle, pancreatic cancer cell lines; closed circle, cancer cell lines from other cancer types). BCRP expression is classified according to SI (SI $>200$, high; $100<$ SI $<200$, moderate; SI $<100$, low). SI of $B C R P$ and derivation of each cancer cell line are shown in Supplemental Table 1.

cancer cell lines displaying varied levels of endogenous BCRP expression. In selecting cancer cell lines, $B C R P$ expression levels in cancer cell lines were classified according to the signal intensity (SI) of $B C R P$ : SI $>200$, high; $100<\mathrm{SI}<200$, moderate; and $\mathrm{SI}<100$, low. Based on this classification, all five cancer cell lines were selected from pancreatic cancer-derived cells to avoid an effect caused by the derivation of tissues. PANC-1 and AsPC-1, HPAF-II and SW 1990, and MIA PaCa-2 were selected as representative cancer cell lines that show high, moderate, and low BCRP expressions, respectively (Fig. 1; Supplemental Table 1). Furthermore, Madin-Darby canine kidney (MDCKII) cells with enforced expression of $B C R P$ were used as a positive control for the experiments.

\section{Materials and Methods}

Reagents. SN-38, etoposide, flavopiridol hydrochloride hydrate, and methotrexate hydrate were purchased from Sigma-Aldrich (St. Louis, MO). Capecitabine was from Wako Pure Chemical Industries (Osaka, Japan). Dinaciclib was from Adooq BioScience (Irvine, CA). Teniposide was from Tokyo Chemical Industry (Tokyo, Japan). Mitoxantrone dihydrochloride was from Abcam Biochemicals (Cambridge, UK). Topotecan hydrochloride was from LKT Laboratories (St. Paul, MN). All other chemicals and reagents were commercial products of reagent grade.

Cell Culture. Human pancreatic cancer cell lines PANC-1, AsPC-1, HPAF-II, SW 1990, and MIA PaCa-2 were obtained from the American Type Culture Collection (Manassas, VA). Parental MDCKII cells were kindly provided by Dr. Piet Borst (Netherlands Cancer Institute, Amsterdam, Netherlands) (Evers et al., 1998). PANC-1, HPAF-II, SW 1990, and MIA $\mathrm{PaCa}-2$ cell lines were grown in high-glucose Dulbecco's modified Eagle's medium supplemented with $10 \%$ fetal bovine serum (FBS). The parental MDCKII cells and their transduced subline, MDCKII-BCRP, were grown in low-glucose Dulbecco's modified Eagle's medium supplemented with 10\% FBS, and AsPC-1 cells were grown in Roswell Park Memorial Institute 1640 medium (Invitrogen, Carlsbad, CA) supplemented with $10 \%$ FBS, in a humidified atmosphere of $5 \% \mathrm{CO}_{2} /$ air at $37^{\circ} \mathrm{C}$.

Transduction of the MDCKII Cell Line with Human BCRP. BCRP CDNA (Matsushima et al., 2005) was subcloned into BamHI and EcoRI sites of the pQCXIN vector (Clontech, Mountain View, CA) to express the target gene along with the neomycin selection marker. Human embryonic kidney 293-T cells were seeded onto collagen-coated culture dishes 1 day before the transfection. BCRP-pQCXIN and
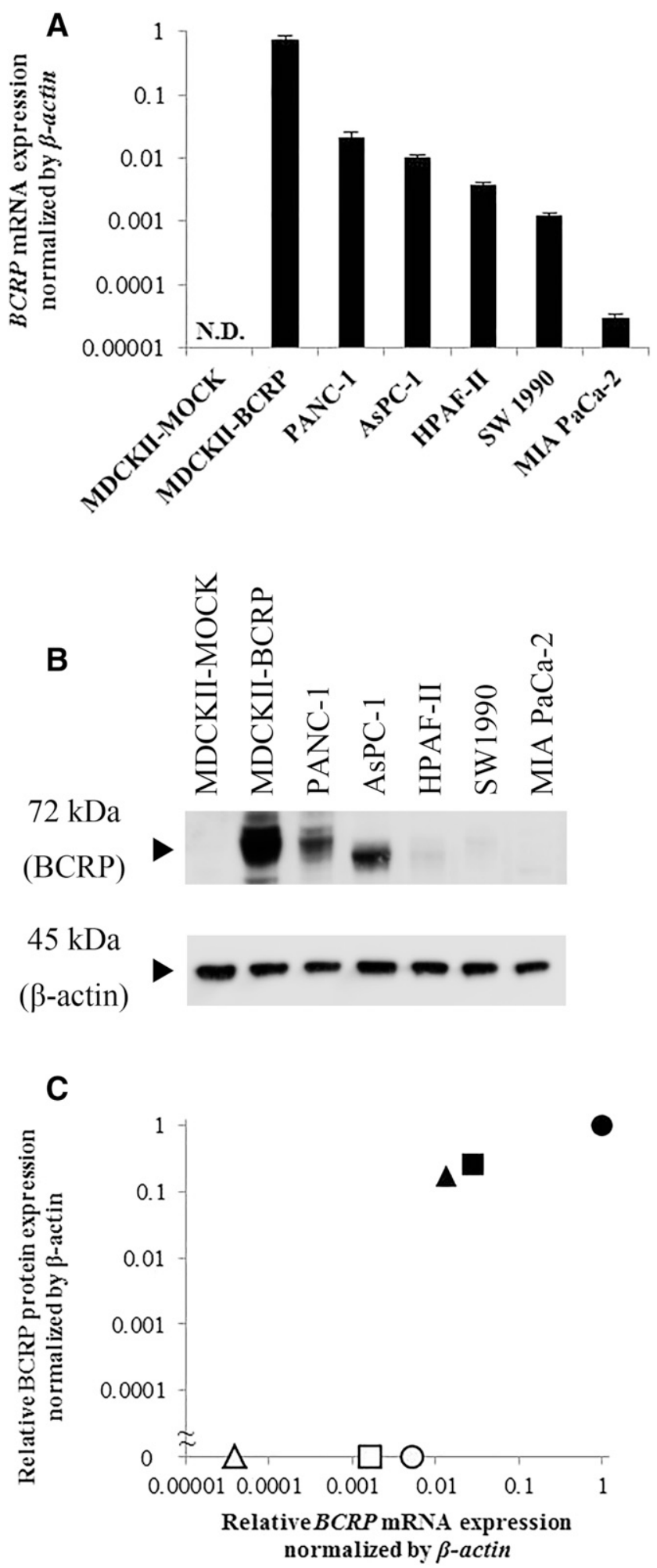

Fig. 2. mRNA (A) and protein (B) expression levels of BCRP in pancreatic cancer cell lines and MDCKII cells and their relationship (C). (A) mRNA expression levels of BCRP were normalized to those of $\beta$-actin as an endogenous control. N.D., BCRP mRNA was not detected. Data represent the mean \pm S.D. $(n=3)$. (B) Ten micrograms (upper) or $5 \mu \mathrm{g}$ (lower) of crude membrane protein was loaded into each well, and BCRP (upper) and $\beta$-actin (lower) proteins were probed with the respective monoclonal antibody. Data are representative of two independent experiments. (C) mRNA and protein expressions of BCRP normalized to those of $\beta$-actin in PANC-1 (closed square), AsPC-1 (closed triangle), HPAF-II (open circle), SW 1990 (open square), and MIA PaCa-2 (open triangle) cells relative to those in MDCKIIBCRP cells (closed circle) are plotted. BCRP protein expression levels were regarded as zero for HPAF-II, SW 1990, and MIA PaCa-2 cells. 
pPAM3psi, which is an amphotropic retrovirus helper plasmid (Miller and Buttimore, 1986), were transfected into human embryonic kidney 293-T cells using FuGENE 6 transfection reagent (Roche Life Science, Penzberg, Germany), following the manufacturer's instructions. After 2 days, virus particles were harvested and concentrated. One day before infection, parental MDCKII cells were seeded onto collagen-coated culture plates. The next day, virus particles collected from the cells transfected with BCRP-pQCXIN were added to parental MDCKII cells with $8 \mu \mathrm{g} / \mathrm{ml}$ of polybrene. Infected cells were selected with G418 (250-500 $\mu \mathrm{g} / \mathrm{ml})$ for BCRP expression. Established cell lines were screened for BCRP activity on the basis of prazosin transport.

TaqMan Reverse-Transcription Polymerase Chain Reaction Gene Expression Analysis and Data Normalization. Total RNA was isolated using the RNeasy Mini Kit (Qiagen, Hilden, Germany). The yield of extracted RNA was measured spectrophotometrically at $260 \mathrm{~nm}$. For first-strand cDNA synthesis,
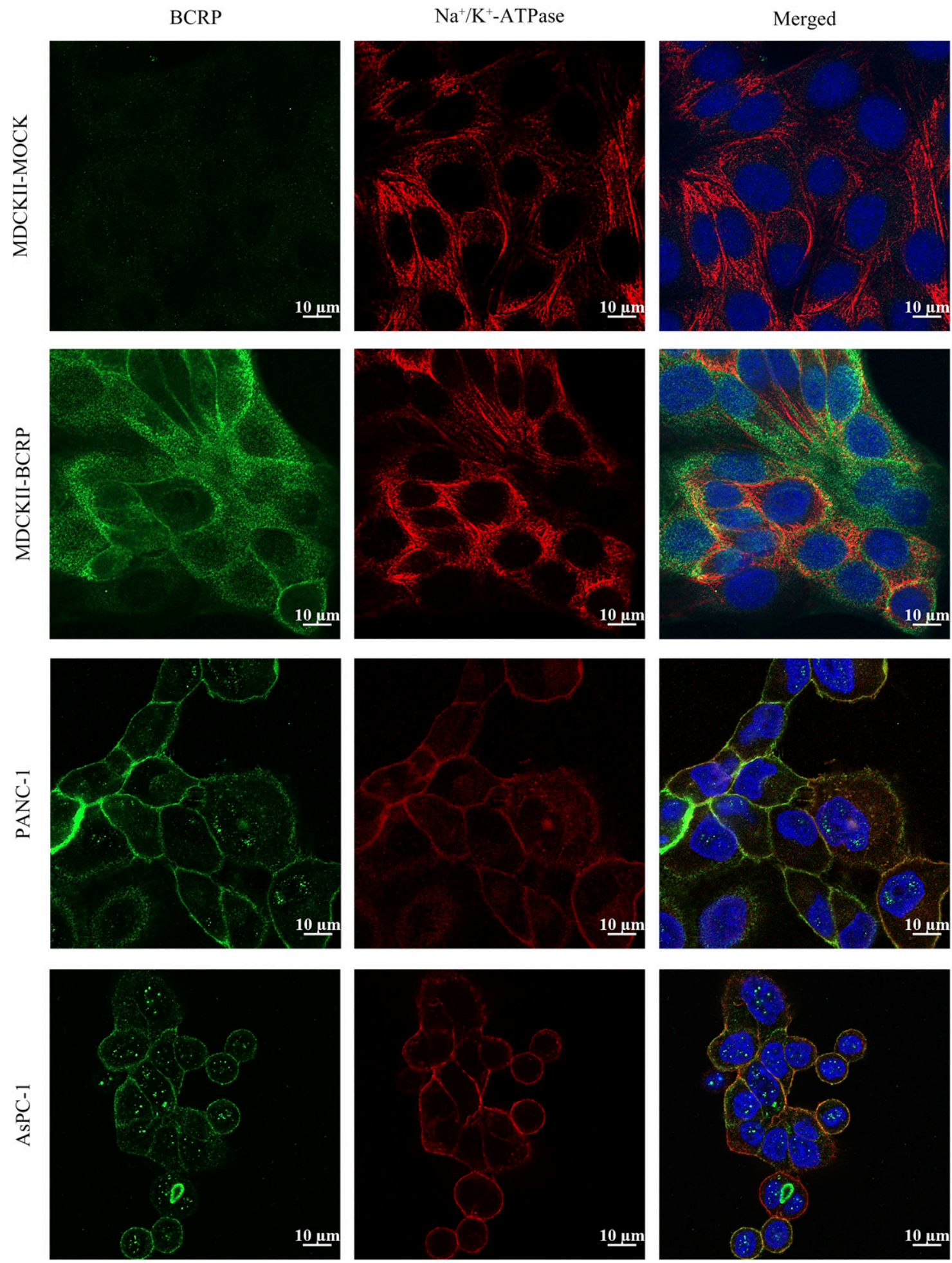

Fig. 3. Confocal immunofluorescence microscopic analysis of BCRP protein localization in pancreatic cancer cell lines and MDCKII cells. Cells were stained with monoclonal antibody against BCRP (green) and polyclonal antibody against $\mathrm{Na}^{+} / \mathrm{K}^{+}$-ATPase (red). Nuclei were stained with DAPI (blue). Images are representative of two independent experiments. 
$500 \mathrm{ng}$ of total RNA was reverse-transcribed in a final volume of $50 \mu \mathrm{l}$ following the protocol for the ReverTra Ace qPCR RT Master Mix (TOYOBO, Osaka, Japan). We used $1 \mu \mathrm{l}$ of cDNA for quantitative real-time polymerase chain reaction (PCR) using the CFX384 Real-Time PCR Detection System (Bio-Rad, Hercules, CA). The assay identification numbers of inventoried TaqMan reversetranscription PCR analysis of human BCRP, human $\beta$-actin, and $\operatorname{dog} \beta$-actin genes were Hs01053790_m1, Hs99999903_m1, and Cf03023880_g1, respectively (Applied Biosystems, Carlsbad, CA). Assays were performed as technical triplicates. Gene expression data were normalized to $\beta$-actin as an endogenous control quantified in the same sample. Relative expression differences were calculated using the comparative $\Delta \Delta \mathrm{C}_{\mathrm{t}}$ method (Livak and Schmittgen, 2001).

Western Blot Analysis. For Western blot analysis, crude membrane fractions were prepared from human pancreatic cancer cell lines and MDCKII cells according to the method described in a previous report (Gant et al., 1991). The protein concentrations in the fraction were determined using the method of Lowry et al. (1951), with bovine serum albumin as a standard. The membrane fraction was dissolved in Laemmli sample buffer with 10\% 2-mercaptoethanol and loaded onto a $10 \%$ Bis-Tris gel. Proteins were transferred electrophoretically to a polyvinylidene difluoride membrane using the Trans-Blot Turbo Transfer System (Bio-Rad) at $1.3 \mathrm{~A}$ for 7 minutes. The membrane was reacted with mouse monoclonal antiBCRP antibody, clone BXP-21 (1:100; KAMIYA Biomedical Company, Seattle, WA), or with rabbit monoclonal anti- $\beta$-actin antibody (1:200; Cell Signaling Technology, Danvers, MA) and then reacted with donkey anti-mouse (for BCRP, 1:500) or donkey anti-rabbit (for $\beta$-actin, 1:2000) IgG conjugated with horseradish peroxidase to detect proteins using the iBind Flex Western Device (Thermo Fisher Scientific, Waltham, MA). Bands were detected using the ECL Prime Western Blotting Detection Reagent (GE Healthcare, Chicago, IL).

Confocal Immunofluorescence Microscopy. Cells grown on glass coverslips were fixed in methanol for 10 minutes at $-20^{\circ} \mathrm{C}$. The cells were permeabilized with $1 \%$ Triton X-100 in phosphate-buffered saline (PBS) for 5 minutes at room temperature. Cells washed with PBS were incubated in blocking solution (5\% FBS in PBS) for 60 minutes and then with mouse monoclonal anti-BCRP antibody, clone BXP-21 (1:25; KAMIYA Biomedical Company), and goat polyclonal anti$\mathrm{Na}^{+} / \mathrm{K}^{+}$-ATPase antibody, N-15 (1:50; Santa Cruz Biotechnology, Dallas, TX). The immunoreactions were visualized by incubation of the cells with donkey Alexa Fluor 488-anti-mouse IgG (for BCRP, 1:1000) and donkey Alexa Fluor 647-anti goat IgG (for $\mathrm{Na}^{+} / \mathrm{K}^{+}$-ATPase, 1:1000). Finally, coverslips containing the immunolabeled cells were mounted with DAPI (Vector Laboratories, Burlingame, CA) and observed using a Zeiss (Oberkochen, Germany) LSM 800. The observations were performed using a Zeiss C-Apochromat $63 \times / 1.2 \mathrm{~W}$ Corr water immersion objective. All images were analyzed using the LSM 800 software.
Cell Proliferation Assay. Cells were seeded onto 96-well plates at a density of 1500 cells/well. On the next day, anticancer drugs were added at different concentrations in the absence or presence of $10 \mu \mathrm{M}$ fumitremorgin C (FTC). After a 72-hour incubation, the cell density was determined using the sulforhodamine $\mathrm{B}$ assay in accordance with reported procedures (Vichai and Kirtikara, 2006). In brief, $10 \%$ trichloroacetic acid was added to each well to fix cells. The plates were washed with water and air-dried, and $0.057 \%$ sulforhodamine B solution was added to each well. Plates were incubated for 30 minutes at room temperature and rinsed with $1 \%$ acetic acid to remove unbound dye. Tris base solution $(10 \mathrm{mM})$ was added to the well to solubilize the protein-bound dye, and optical density (OD) at $540 \mathrm{~nm}$ was measured using a microplate reader. Assays were performed as technical triplicates.

mRNA Quantification after Treatment with Anticancer Drugs. Cells were seeded into 24-well plates at a density of 8900 cells/well. The next day, the cells were exposed to anticancer drugs at the indicated concentrations for 72 hours. RNA isolation, reverse transcription, and quantitative real-time PCR were performed as described in the previous section.

Data Analysis. The percentage of control cell growth was calculated using the following equation:

$$
\% \text { of control cell growth }=\frac{\mathrm{OD}_{\text {sample }}-\mathrm{OD}_{\text {day } 0}}{\mathrm{OD}_{\text {neg control }}-\mathrm{OD}_{\text {day } 0}}
$$

where $\mathrm{OD}_{\text {sample }}$ and $\mathrm{OD}_{\text {neg control }}$ are mean $\mathrm{ODs}$ of the cells with and without anticancer drugs, respectively, and $\mathrm{OD}_{\text {day0 }}$ is the mean $\mathrm{OD}$ of the cells before anticancer drugs were added.

The concentration that induces $50 \%$ of the maximum effect on cell growth inhibition $\left(\mathrm{EC}_{50}\right)$ of anticancer drugs tested was determined by iterative nonlinear regression analysis using Excel (Microsoft, Redmond, WA) and its add-in program XLfit (IDBS, Guildford, UK). EC $_{50}$ values were calculated, assuming standard hyperbolic kinetics, in accordance with the following equation:

$$
E=E_{\min }+\left[\frac{E_{\max }-E_{\min }}{1+\left(\frac{x}{E \mathrm{C}_{50}}\right)^{H}}\right]
$$

where $x$ is the drug concentration; $E_{\min }$ and $E_{\max }$ are the percentages of cell growth when $x$ is infinity and 0 , respectively; and $H$ is the slope factor.

\section{Results}

mRNA and Protein Expression of BCRP in Pancreatic Cancer Cell Lines and MDCKII Cells. $B C R P$ mRNA expression levels in five

\section{TABLE 1}

$\mathrm{EC}_{50}$ values of anticancer drugs that are substrates of BCRP in MDCKII-MOCK and MDCKII-BCRP cells

For each drug and cell type, the table displays the median $E C_{50}$ value, range of $E C_{50}$ (micromolars), number of experimental determinations of $E C_{50}$ performed ( $N$ ), and resistance ratio $(R R)$. $R R$ was calculated by dividing the median $E C_{50}$ for a given drug in the absence of $F T C$ ( $\left.E C_{50},-F T C\right)$ by the median $E C_{50}$ of that drug in the

\begin{tabular}{|c|c|c|c|c|c|c|c|c|}
\hline \multirow{2}{*}{ Drug } & \multicolumn{4}{|c|}{ MDCKII-MOCK } & \multicolumn{4}{|c|}{ MDCKII-BCRP } \\
\hline & $\mathrm{EC}_{50,-\mathrm{FTC}}$ (range) & $\mathrm{EC}_{50,+\mathrm{FTC}}$ (range) & $\mathrm{N}$ & $\mathrm{RR}$ & $\mathrm{EC}_{50,-\mathrm{FTC}}$ (range) & $\mathrm{EC}_{50,+\mathrm{FTC}}$ (range) & $\mathrm{N}$ & RR \\
\hline & $\mu M$ & $\mu M$ & & & $\mu M$ & $\mu M$ & & \\
\hline \multicolumn{9}{|l|}{ Cytotoxic } \\
\hline \multicolumn{9}{|l|}{ Antimetabolites } \\
\hline Capecitabine & $>200(-)$ & $>200(-)$ & 1 & $\mathrm{NC}$ & $>200(-)$ & $>200(-)$ & 1 & $\mathrm{NC}$ \\
\hline Methotrexate & $0.035(-)$ & $0.028(-)$ & 1 & 1.3 & $0.047(-)$ & $0.026(-)$ & 1 & 1.8 \\
\hline \multicolumn{9}{|c|}{ Topoisomerase I inhibitor } \\
\hline SN-38 & $0.16(0.14-0.17)$ & $0.051(0.044-0.057)$ & 2 & 3.1 & $6.5(6.4-6.6)$ & $0.070(0.069-0.070)$ & 2 & 94 \\
\hline Topotecan & $0.39(0.31-0.46)$ & $0.26(0.19-0.33)$ & 2 & 1.5 & $14(12-16)$ & $0.44(0.36-0.52)$ & 2 & 32 \\
\hline \multicolumn{9}{|c|}{ Topoisomerase II inhibitor } \\
\hline Mitoxantrone & $0.29(0.29-0.29)$ & $0.26(0.26-0.26)$ & 2 & 1.1 & $1.2(1.1-1.3)$ & $0.29(0.28-0.30)$ & 3 & 4.0 \\
\hline Etoposide & $2.8(-)$ & $1.8(-)$ & 1 & 1.6 & $5.2(-)$ & $2.1(-)$ & 1 & 2.5 \\
\hline Teniposide & $0.57(-)$ & $0.42(-)$ & 1 & 1.4 & $1.4(-)$ & $0.47(-)$ & 1 & 3.0 \\
\hline \multicolumn{9}{|l|}{ Molecular targeted } \\
\hline \multicolumn{9}{|c|}{ Cyclin-dependent kinase inhibitor } \\
\hline Dinaciclib & $0.065(-)$ & $0.030(-)$ & 1 & 2.2 & $0.18(-)$ & $0.027(-)$ & 1 & 6.7 \\
\hline Flavopiridol & $0.12(0.12-0.12)$ & $0.12(0.11-0.12)$ & 2 & 1.0 & $0.54(0.46-0.61)$ & $0.097(0.084-0.11)$ & 2 & 5.5 \\
\hline
\end{tabular}
presence of FTC $\left(E C_{50},+F T C\right)$ for each cell type.

NC, not calculated. 
pancreatic cancer cell lines and MDCKII cells were determined using TaqMan real-time PCR analysis and normalized to $\beta$-actin expression as an endogenous control. The relative mRNA expression level of $B C R P$ was the highest in MDCKII-BCRP cells, followed by PANC-1, AsPC-1, HPAF-II, SW 1990, and MIA PaCa-2 cells. BCRP mRNA was not detected in MDCKII-MOCK cells (Fig. 2A). The experimentally determined $B C R P$ mRNA expression ratio in five pancreatic cancer cell lines was comparable to that from microarray data in CCLE (data not shown). The mRNA expression levels of $P$ - $g p$, which also confers multidrug resistance, were also quantified in pancreatic cancer cell lines. The mRNA expression levels of $P$ - $g p$ were below the detection limit in AsPC-1, SW 1990, and MIA PaCa-2 cells or more than 6900-fold lower than those of $\beta$-actin in PANC-1 and HPAF-II cells, suggesting that $P$ $g p$ expression in these cell lines was very low (data not shown). The expression levels of BCRP protein in the crude membrane fraction prepared from pancreatic cancer cell lines and MDCKII cells were investigated by Western blot analysis. BCRP protein was detected in MDCKII-BCRP, PANC-1, and AsPC-1 cells but not in MDCKIIMOCK, HPAF-II, SW 1990, and MIA PaCa-2 cells (Fig. 2B; Supplemental Fig. 1A). The protein molecular weight of BCRP in AsPC-1 cells was lower than that in MDCKII-BCRP or PANC-1 cells. The relative expression level of BCRP protein normalized to $\beta$-actin protein became larger with an increase of $B C R P$ mRNA expression in MDCKII-BCRP, PANC-1, and AsPC-1 cells (Fig. 2C).

Subcellular Localization of the BCRP Protein in Pancreatic Cancer Cell Lines and MDCKII Cells. Because the BCRP protein was detected in Western blot analysis in MDCKII-BCRP, PANC-1, and AsPC-1 cells (Fig. 2B), the subcellular localization of the BCRP protein was examined in these cells using confocal immunofluorescence microscopic analysis. The $\mathrm{Na}^{+} / \mathrm{K}^{+}$-ATPase protein was costained with BCRP as a marker of the plasma membrane. In MDCKII-BCRP cells, the BCRP protein was expressed not only in the plasma membrane but also throughout the cells, and a stronger signal could be observed in the plasma membrane than inside the cells (Fig. 3). The BCRP protein was not detected in MDCKII-MOCK cells (Fig. 3). In PANC-1 and AsPC-1 cells, BCRP was expressed mainly in the plasma membranes (Fig. 3).

$\mathbf{E C}_{50}$ Values of Anticancer Drugs in the Absence and Presence of FTC in MDCKII Cells. Nine anticancer drugs that are substrates of BCRP and have different modes of action were selected (Table 1), and $\mathrm{EC}_{50}$ values of these drugs were evaluated in the absence and presence of a BCRP inhibitor, FTC, in MDCKII-MOCK and MDCKII-BCRP cells. FTC was used as a selective BCRP inhibitor (Ahmed-Belkacem et al., 2006) to evaluate the impact of only BCRP inhibition. Resistance ratio (RR) was calculated based on the ratio of $\mathrm{EC}_{50}$ for a given drug without FTC over that with FTC in each cell line. Methotrexate, SN-38, topotecan, mitoxantrone, etoposide, teniposide, dinaciclib, and flavopiridol inhibited the growth of MDCKII-MOCK and MDCKII-BCRP cells in a concentration-dependent manner, whereas capecitabine did not inhibit the growth of MDCKII-MOCK and MDCKII-BCRP cells up to $200 \mu \mathrm{M}$. Although RRs of SN-38 (3.1) and dinaciclib (2.1) were $>2$, RRs of most anticancer drugs were $<2$ in MDCKII-MOCK cells in which BCRP was not expressed (Table 1). Therefore, RR $>2$ was considered to be a significant effect by BCRP inhibition. RR of methotrexate was $<2$, and RRs of SN-38, topotecan, mitoxantrone, etoposide, teniposide, dinaciclib, and flavopiridol in MDCKII-BCRP cells were > 2 (94, 32, 4.0, 2.5, 3.0, 6.7, and 5.5, respectively). By the addition of $\mathrm{FTC}, \mathrm{EC}_{50}$ values of these drugs in MDCKII-BCRP cells decreased to values comparable to those of MDCKII-MOCK cells, which suggested that FTC completely inhibited BCRP activity in MDCKII-BCRP cells (Table 1). Among nine anticancer drugs investigated, $\mathrm{EC}_{50}$ values of $\mathrm{SN}-38$, topotecan, mitoxantrone, dinaciclib, and flavopiridol were further determined in

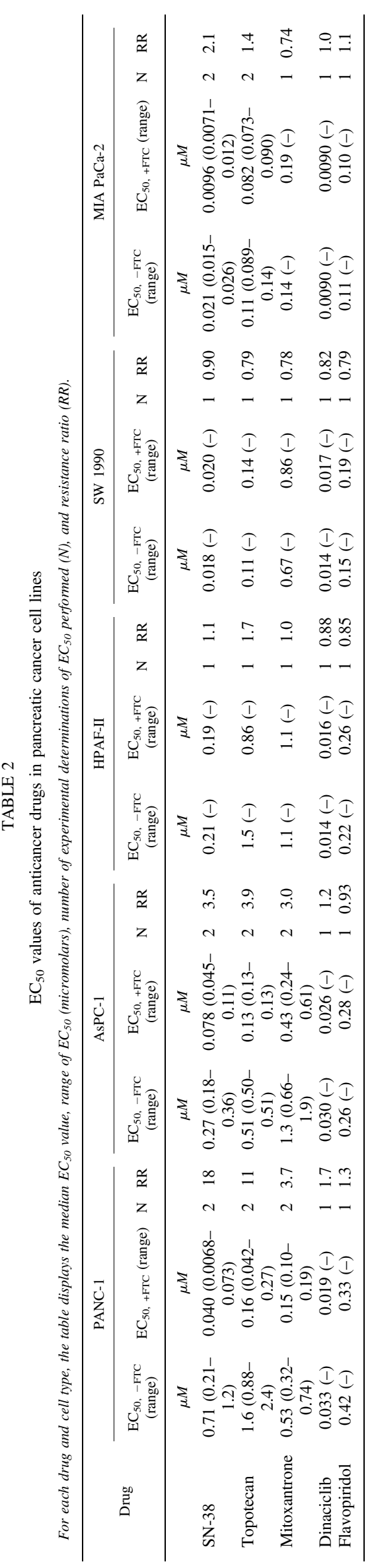


the selected pancreatic cancer cell lines because these drugs showed larger RRs in MDCKII-BCRP cells.

EC $_{50}$ Values of Anticancer Drugs in the Absence and Presence of FTC in Selected Cancer Cell Lines. EC $_{50}$ values of SN-38, topotecan, mitoxantrone, dinaciclib, and flavopiridol in the absence and presence of FTC were evaluated in five pancreatic cancer cell lines, and RRs were calculated. RRs of SN-38, topotecan, and mitoxantrone were 18, 11, and 3.7 , respectively, in PANC-1 and 3.5, 3.9, and 3.0, respectively, in AsPC-1 (Table 2). RRs of dinaciclib and flavopiridol in PANC-1 and AsPC-1 cells were <2. In HPAF-II, SW 1990, and MIA PaCa- 2 cells, none of these five drugs showed RRs $>2$, except SN-38 in MIA PaCa-2 cells (Table 2).
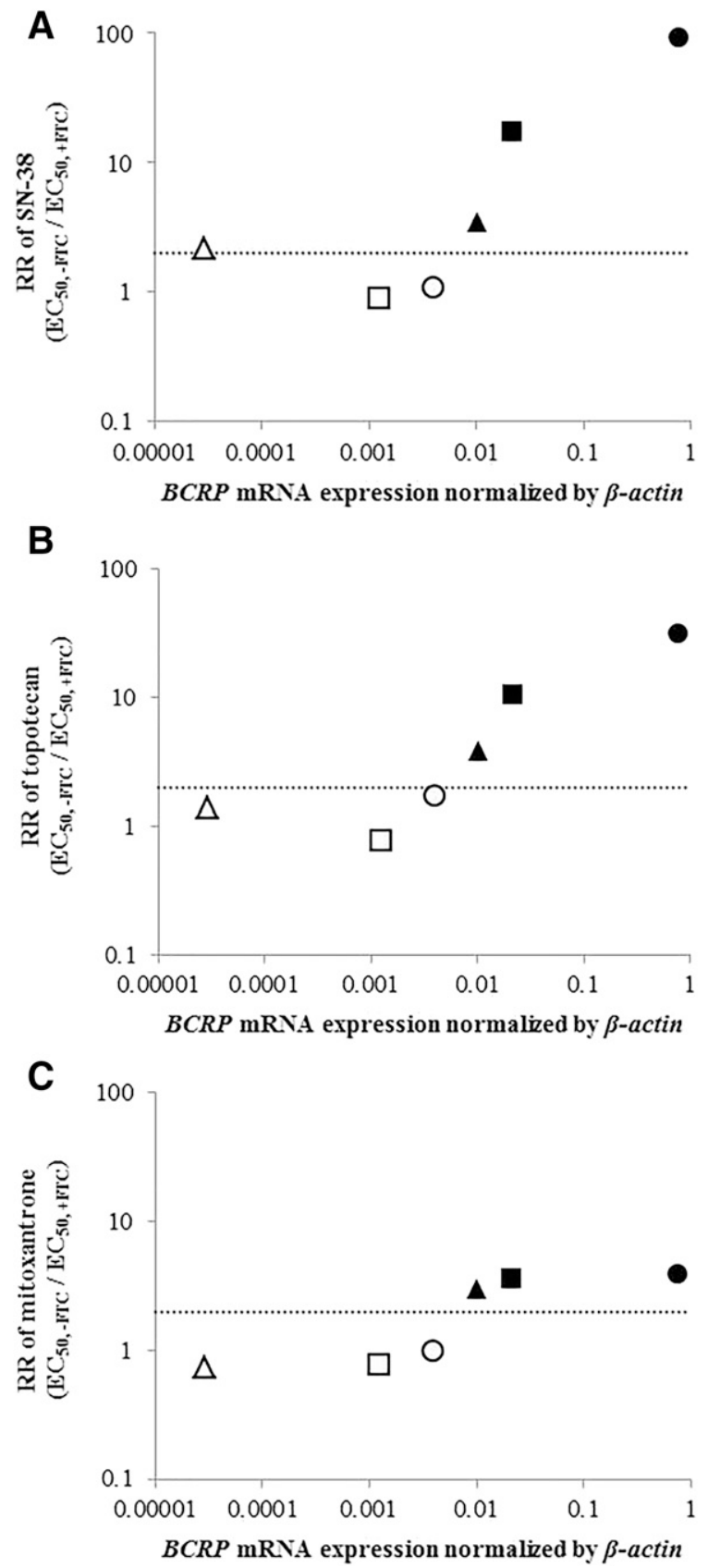

Relationship between RRs of Anticancer Drugs and BCRP Expression in Pancreatic Cancer Cell Lines and MDCKII-BCRP Cells. The relationship between RRs of SN-38, topotecan, mitoxantrone, dinaciclib, and flavopiridol and mRNA expression levels of $B C R P$ in five pancreatic cancer cell lines and MDCKIIBCRP cells was examined. The results are shown in Fig. 4. RRs of all these drugs increased with an increase in BCRP mRNA expression.

Effect of Drug Treatment on BCRP mRNA Expression in Pancreatic Cancer Cell Lines. Effect of anticancer drugs on BCRP mRNA expression was examined in pancreatic cancer cell lines after treatment with SN-38, topotecan, mitoxantrone, dinaciclib, and
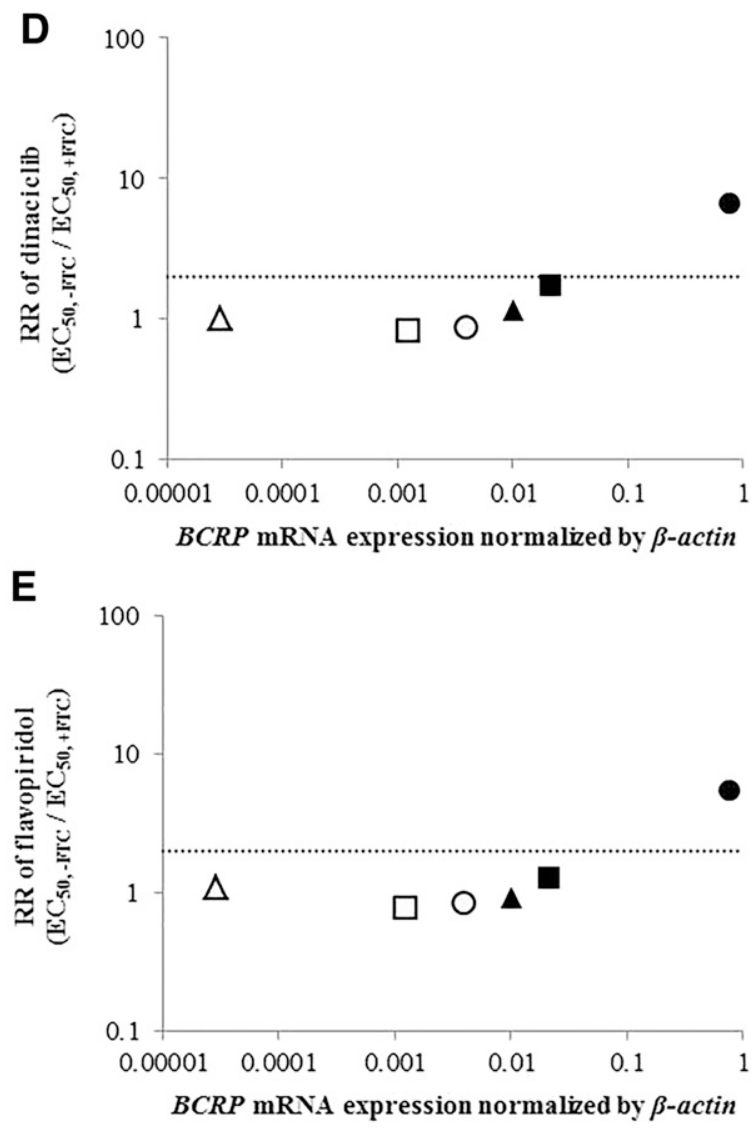

Fig. 4. Relationship between RR of SN-38 (A), topotecan (B), mitoxantrone (C), dinaciclib (D), and flavopiridol (E) and BCRP mRNA expression levels of MDCKII-BCRP (closed circle), PANC-1 (closed square), AsPC-1 (closed triangle), HPAF-II (open circle), SW 1990 (open square), and MIA PaCa-2 (open triangle) cells. Dotted lines represent an RR of 2. 
flavopiridol at concentrations close to $\mathrm{EC}_{50}$ values for 72 hours. In PANC-1, AsPC-1, HPAF-II, and SW 1990 cells, alterations in BCRP mRNA expression after treatment with anticancer drugs were within 2-fold. In MIA PaCa-2 cells, a concentration-dependent, >10-fold increase in $B C R P$ mRNA expression was observed after treatment with $\mathrm{SN}-38$, topotecan, and mitoxantrone, with increases in expression at higher concentrations of 15-, 17-, and 20-fold, respectively (Table 3).

\section{Discussion}

In CCLE, $28 \%$ pancreatic cancer cell lines (12 of 43) express BCRP at high levels, and the rest of them show moderate or low $B C R P$ expression based on the classification of SI (SI $>200$, high; $100<$ SI $<200$, moderate; and SI $<100$, low) (Fig. 1). In the present study, we investigated the impact of BCRP expression on the efficacy of anticancer drugs that are BCRP substrates in the presence or absence of a selective BCRP inhibitor, using several pancreatic cancer cell lines with varying levels of BCRP expression. Our results show that the $\mathrm{EC}_{50}$ values of some anticancer drugs significantly decrease in the presence of a BCRP inhibitor in PANC-1 and AsPC-1 cells expressing high levels of BCRP. The largest shift of $\mathrm{EC}_{50}$ observed in the present study was 18-fold, in

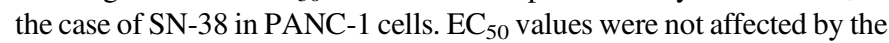
BCRP inhibitor in HPAF-II, SW 1990, and MIA PaCa-2 cells expressing moderate or low levels of BCRP (Table 2). Overall, RRs of anticancer drugs became larger with an increase in BCRP mRNA expression (Fig. 4). Because all cell lines used in this study were derived from pancreatic cancer, further investigations are required to evaluate whether the findings are true in cell lines derived from other cancer types.

Because it is well recognized that mRNA expression is a surrogate of protein expression, and that transporters are functional when expressed in plasma membranes of cells, a relationship between mRNA (Fig. 2A), protein expression (Fig. 2B; Supplemental Fig. 1A), and protein subcellular localization (Fig. 3) was examined. In HPAF-II, SW 1990, and MIA PaCa- 2 cells, BCRP protein was not detected despite $B C R P$ mRNA expression being detected in significant levels. This can be attributed to the higher sensitivity of real-time PCR compared with that of Western blot analysis. Considering robust BCRP protein expression in plasma membranes of PANC-1 and AsPC-1 cells, BCRP mRNA expression levels might serve as a good indicator for functional BCRP, at least in PANC-1 and AsPC-1 cells. In Western blot analysis, the protein molecular weight of BCRP in AsPC-1 cells was less than that in
MDCKII-BCRP or PANC-1 cells (Fig. 2B; Supplemental Fig. 1A). This observation might be accounted for by the difference in $N$-linked glycosylation of BCRP between AsPC-1 and MDCKII-BCRP cells or PANC-1 cells. However, the difference may not have a significant impact on BCRP function because the absence of $N$-linked glycosylation did not affect the plasma membrane localization of BCRP (Mohrmann et al., 2005). In fact, RRs of SN-38, topotecan, and mitoxantrone were $>2$ in AsPC-1 cells, which suggested that BCRP expressed in AsPC-1 cells was functional.

$B C R P$ mRNA expression increased in the MIA PaCa- 2 cells treated with anticancer drugs (Table 3); however, the increased expression levels in these cells still remained the lowest in rank order among the five pancreatic cancer cell lines used in this study. Thus, no changes in BCRP mRNA expression occurred after incubation with anticancer drugs, which affected RRs of these drugs and interpretation of the results shown in Fig. 4. Because the number of cell lines used in this study was limited, further investigations are required to identify any cancer cell lines that show increased BCRP expression sufficient to alter RRs after treatment with anticancer drugs.

As shown in Fig. 4, the overall RRs of anticancer drugs could be explained by BCRP mRNA expression levels. However, in the present study, RR of SN-38 was 2.1 in MIA PaCa- 2 cells, which was of borderline significance for the BCRP effect despite low BCRP expression (Table 2), and such a high RR value of SN-38 was also observed in MDCKII-MOCK cells (3.1) (Table 1). SN-38 was reported to be a substrate of not only BCRP but also other efflux transporters, such as P-gp, multidrug-resistance protein 1, and multidrug-resistance protein 2 (Kwak et al., 2012). Because the mRNA expression level of $P$ $g p$ in MIA PaCa- 2 cells was low, endogenous transporters other than BCRP and P-gp, whose function can be altered by FTC, may be involved in this resistance.

Transcellular transport experiments of SN-38, topotecan, mitoxantrone, and flavopiridol using MDCKII-MOCK and MDCKII-BCRP cells have been reported (Xiao et al., 2006; Zhou et al., 2009), and the corrected efflux ratio, which is the normalized ratio of basal-to-apical versus apical-to-basal permeability in MDCKII-MOCK cell monolayers with respect to that in MDCKII-BCRP cell monolayers, of these four drugs was comparable (7.6 8.4 in $\mathrm{SN}-38,2.6 \sim 5.4$ in topotecan, $4.5 \sim 6.1$ in mitoxantrone, and 6.0 in flavopiridol). However, our study showed that RRs of topoisomerase I inhibitors (SN-38 and topotecan) were much larger than those of mitoxantrone and flavopiridol in MDCKII-BCRP cells (Table 1). The magnitude of RRs may not be

TABLE 3

Relative changes in BCRP mRNA expression in pancreatic cancer cell lines after treatment with anticancer drugs compared with control (no treatment) Analysis of mRNA expression was performed after a 72-hour incubation with each anticancer drug. BCRP mRNA expression levels were normalized to those of $\beta$-actin. Data represent the mean \pm S.D. $(n=3)$.

\begin{tabular}{|c|c|c|c|c|c|c|c|c|c|c|}
\hline \multirow{2}{*}{ Drug } & \multicolumn{2}{|c|}{ PANC-1 } & \multicolumn{2}{|c|}{ AsPC-1 } & \multicolumn{2}{|c|}{ HPAF-II } & \multicolumn{2}{|c|}{ SW 1990} & \multicolumn{2}{|c|}{ MIA $\mathrm{PaCa}-2$} \\
\hline & Concentration & Mean \pm S.D. & Concentration & Mean \pm S.D. & Concentration & Mean \pm S.D. & Concentration & Mean \pm S.D. & Concentration & Mean \pm S.D. \\
\hline \multirow[b]{2}{*}{ Control } & $\mu \mathrm{M}$ & & $\mu \mathrm{M}$ & & $\mu \mathrm{M}$ & & $\mu \mathrm{M}$ & & $\mu \mathrm{M}$ & \\
\hline & - & $1.0 \pm 0.056$ & - & $1.0 \pm 0.032$ & - & $1.0 \pm 0.12$ & - & $1.0 \pm 0.13$ & - & $1.0 \pm 0.19$ \\
\hline \multirow{2}{*}{ SN-38 } & 0.3 & $1.8 \pm 0.18$ & 0.1 & $1.4 \pm 0.34$ & 0.1 & $0.76 \pm 0.053$ & 0.01 & $1.3 \pm 0.17$ & 0.01 & $8.3 \pm 1.4$ \\
\hline & 1 & $1.8 \pm 0.28$ & 0.3 & $0.94 \pm 0.15$ & 0.3 & $1.2 \pm 0.25$ & 0.03 & $1.0 \pm 0.096$ & 0.03 & $15 \pm 1.7$ \\
\hline \multirow{2}{*}{ Topotecan } & 1 & $1.7 \pm 0.24$ & 0.3 & $1.5 \pm 0.070$ & 0.3 & $0.76 \pm 0.044$ & 0.03 & $1.4 \pm 0.11$ & 0.03 & $3.1 \pm 0.19$ \\
\hline & 3 & $1.8 \pm 0.18$ & 1 & $0.75 \pm 0.097$ & 1 & $0.83 \pm 0.080$ & 0.1 & $0.85 \pm 0.14$ & 0.1 & $17 \pm 0.89$ \\
\hline \multirow{2}{*}{ Mitoxantrone } & 0.1 & $2.0 \pm 0.15$ & 0.3 & $1.3 \pm 0.20$ & 0.3 & $0.65 \pm 0.090$ & 0.1 & $0.70 \pm 0.14$ & 0.03 & $17 \pm 1.8$ \\
\hline & 0.3 & $1.8 \pm 0.14$ & 1 & $0.75 \pm 0.089$ & 1 & $1.2 \pm 0.20$ & 0.3 & $1.1 \pm 0.14$ & 0.1 & $20 \pm 1.4$ \\
\hline \multirow{2}{*}{ Dinaciclib } & 0.01 & $0.73 \pm 0.018$ & 0.01 & $0.98 \pm 0.17$ & 0.003 & $0.78 \pm 0.040$ & 0.003 & $0.76 \pm 0.055$ & 0.003 & $1.2 \pm 0.17$ \\
\hline & 0.03 & $1.9 \pm 0.13$ & 0.03 & $0.99 \pm 0.087$ & 0.01 & $1.2 \pm 0.057$ & 0.01 & $1.3 \pm 0.19$ & 0.01 & $4.1 \pm 0.45$ \\
\hline \multirow{2}{*}{ Flavopiridol } & 0.1 & $0.65 \pm 0.094$ & 0.1 & $0.97 \pm 0.10$ & 0.1 & $1.2 \pm 0.11$ & 0.03 & $0.71 \pm 0.10$ & 0.03 & $0.99 \pm 0.085$ \\
\hline & 0.3 & $1.2 \pm 0.17$ & 0.3 & $1.7 \pm 0.31$ & 0.3 & $1.5 \pm 0.13$ & 0.1 & $0.76 \pm 0.12$ & 0.1 & $1.7 \pm 0.25$ \\
\hline
\end{tabular}


directly explained by the efflux ratio alone, which is an indicator of the importance of efflux transporters in transporting drugs across the cell membrane (Giacomini et al., 2010). Namely, there may be a determinant rather than the active efflux of anticancer drugs by BCRP. For example, the anticancer efficacy of drugs depends on their chemical structure within the tumor microenvironment. SN-38 and topotecan are pharmacologically active in the lactone form, but most drugs are in the inactive carboxylate form at physiologic $\mathrm{pH}$ (Oguma, 2001). With reference to the transport of lactone and carboxylate forms of $\mathrm{SN}-38$, we have recently shown that they are similarly transported by another type of transporter, organic anion-transporting polypeptide 2B1 (Fujita et al., 2016). However, the same result may not be directly applicable to BCRP-mediated transport, and evaluation of the efflux ratio of the lactone forms of these drugs separately from their carboxylate forms will be required.

$B C R P$ mRNA expression in pancreatic cancer cell lines used in this study was compared with that in cancer biopsies available from the Expression Project for Oncology (accession number: GSE2109; Gene Expression Omnibus), which provides public access to mRNA expression data of more than 2000 cancer biopsies (Fig. 5; Supplemental Table 2). The comparison showed that mRNA expression levels of $B C R P$ in cancer biopsies were lower than those in PANC-1 and AsPC-1 cells, in which significant decreases in $\mathrm{EC}_{50}$ values of several anticancer drugs were observed when BCRP was inhibited. BCRP mRNA expression in cancer biopsies was within a comparable range or lower than that in HPAF-II, SW 1990, and MIA PaCa-2 cells, in which significant alterations in $\mathrm{EC}_{50}$ values were not observed (Fig. 5; Supplemental Table 2). However, this does not necessarily mean that drug resistance caused by BCRP-mediated efflux in cancer tissues can be ignored. Although $B C R P$ mRNA expression increased in MIA PaCa2 cells exposed to anticancer drugs (Table 3 ) and in vivo, leukemic blasts from patients with acute leukemia showed a significant increase in $B C R P$ mRNA expression after in vivo flavopiridol exposure (Nakanishi et al., 2003b); more than $90 \%$ of the cancer biopsies shown in Fig. 5 were from patients who did not receive any chemotherapy before surgical resection, according to clinically annotated Expression Project for Oncology information (accession number: GSE2109; Gene Expression Omnibus). Therefore, it should be kept in mind that $B C R P$ expression levels in cancer tissues after chemotherapy might be different from those shown in Fig. 5. In addition, cancer tissues consist of heterogeneous

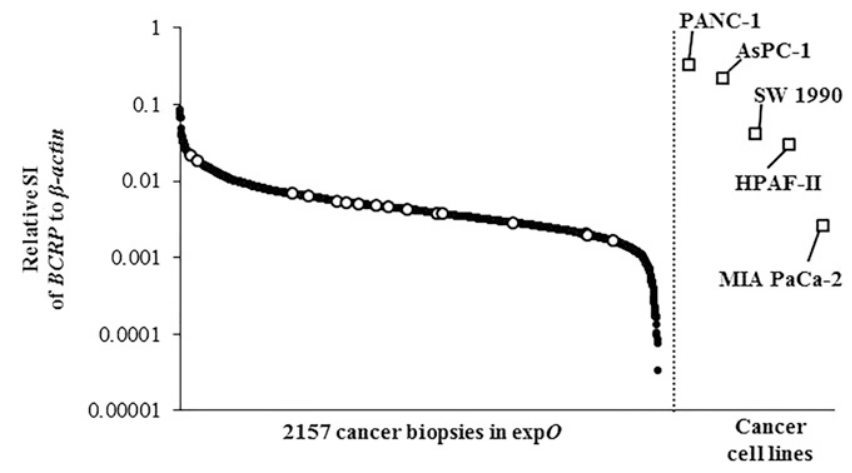

Fig. 5. Comparison of relative mRNA expression levels of $B C R P$ to $\beta$-actin between cancer biopsies and cancer cell lines. mRNA expression levels of BCRP and $\beta$-actin in 2157 cancer biopsies and cancer cell lines used in this study, represented as SI of microarray analysis, were obtained from Expression Project for Oncology $(\exp O)$ and CCLE, respectively, and compared (open circle, pancreatic cancer biopsies; closed circle, cancer biopsies derived from malignant tissues other than pancreas; open square, pancreatic cancer cell lines). SI of BCRP and $\beta$-actin, relative SI of $B C R P$ to $\beta$-actin, and derivation of each cancer biopsy is shown in Supplemental Table 2. cells, and BCRP is reported to be highly expressed in cancer stem cells that are considered responsible for the tumorigenic process (An and Ongkeko, 2009; Natarajan et al., 2012). However, BCRP expression levels in cancer biopsies shown in Fig. 5 represent an average across heterogeneous cells, including cancer stem cells and other types of cells.

In conclusion, our results suggest that the in vitro efficacy of anticancer drugs on cell proliferation was significantly affected by BCRP in pancreatic cancer cell lines abundantly expressing BCRP, which account for $28 \%$ pancreatic cancer cell lines in CCLE. However, the in vitro efficacy of anticancer drugs in the remaining $72 \%$ of pancreatic cancer cell lines expressing BCRP at moderate or low levels was not significantly affected. Although we are unable to exclude the possibility that BCRP expression induced by anticancer drug treatment during incubation affected their in vitro efficacy, it is unlikely that clinically promising anticancer drugs for treatment of pancreatic cancer have been excluded during screening because of BCRP, considering that most pancreatic cancer cell lines show moderate or low BCRP expression. However, when pancreatic cancer cell lines with high levels of BCRP expression are used, the effect of BCRP should be carefully evaluated, considering information such as BCRP expression in cancer cell lines and cancer tissues from patients, to avoid errors in the selection of drug candidates.

\section{Acknowledgments}

We thank Asami Saito (Nippon Boehringer Ingelheim Co., Ltd.) for preparing MDCKII-BCRP cells.

\section{Authorship Contributions}

Participated in research design: Washio, Nakanishi, Ishiguro, Yamamura, Tamai.

Conducted experiments: Washio.

Performed data analysis: Washio, Nakanishi, Ishiguro, Tamai.

Wrote or contributed to the writing of the manuscript: Washio, Nakanishi, Ishiguro, Tamai.

\section{References}

Ahmed-Belkacem A, Pozza A, Macalou S, Pérez-Victoria JM, Boumendjel A, and Di Pietro A (2006) Inhibitors of cancer cell multidrug resistance mediated by breast cancer resistance protein (BCRP/ABCG2). Anticancer Drugs 17:239-243.

Allen JD, Van Dort SC, Buitelaar M, van Tellingen O, and Schinkel AH (2003) Mouse breast cancer resistance protein (Bcrp1/Abcg2) mediates etoposide resistance and transport, but etoposide oral availability is limited primarily by P-glycoprotein. Cancer Res 63:1339-1344. An Y and Ongkeko WM (2009) ABCG2: the key to chemoresistance in cancer stem cells? Expert Opin Drug Metab Toxicol 5:1529-1542.

Barretina J, Caponigro G, Stransky N, Venkatesan K, Margolin AA, Kim S, Wilson CJ, Lehár J, Kryukov GV, Sonkin D, et al. (2012) The cancer cell line encyclopedia enables predictive modelling of anticancer drug sensitivity. Nature 483:603-607.

Burger H, van Tol H, Boersma AW, Brok M, Wiemer EA, Stoter G, and Nooter K (2004) Imatinib mesylate (STI571) is a substrate for the breast cancer resistance protein (BCRP)/ABCG2 drug pump. Blood 104:2940-2942.

Cihalova D, Ceckova M, Kucera R, Klimes J, and Staud F (2015) Dinaciclib, a cyclin-dependent kinase inhibitor, is a substrate of human ABCB1 and ABCG2 and an inhibitor of human ABCC1 in vitro. Biochem Pharmacol 98:465-472.

Doyle LA, Yang W, Abruzzo LV, Krogmann T, Gao Y, Rishi AK, and Ross DD (1998) A multidrug resistance transporter from human MCF-7 breast cancer cells. Proc Natl Acad Sci USA 95:15665-15670.

Edmondson R, Broglie JJ, Adcock AF, and Yang L (2014) Three-dimensional cell culture systems and their applications in drug discovery and cell-based biosensors. Assay Drug Dev Technol 12 : $207-218$.

Evers R, Kool M, van Deemter L, Janssen H, Calafat J, Oomen LC, Paulusma CC, Oude Elferink RP, Baas F, Schinkel AH, et al. (1998) Drug export activity of the human canalicular multispecific organic anion transporter in polarized kidney MDCK cells expressing cMOAT (MRP2) cDNA. J Clin Invest 101:1310-1319.

Fujita D, Saito Y, Nakanishi T, and Tamai I (2016) Organic Anion Transporting Polypeptide (OATP)2B1 contributes to gastrointestinal toxicity of anticancer drug SN-38, active metabolite of irinotecan hydrochloride. Drug Metab Dispos 44:1-7.

Gant TW, Silverman JA, Bisgaard HC, Burt RK, Marino PA, and Thorgeirsson SS (1991) Regulation of 2-acetylaminofluorene-and 3-methylcholanthrene-mediated induction of multidrug resistance and cytochrome P450IA gene family expression in primary hepatocyte cultures and rat liver. Mol Carcinog 4:499-509.

Giacomini KM, Huang SM, Tweedie DJ, Benet LZ, Brouwer KL, Chu X, Dahlin A, Evers R, Fischer V, Hillgren KM, et al.; International Transporter Consortium (2010) Membrane transporters in drug development. Nat Rev Drug Discov 9:215-236. 
Kwak EY, Shim WS, Chang JE, Chong S, Kim DD, Chung SJ, and Shim CK (2012) Enhanced intracellular accumulation of a non-nucleoside anti-cancer agent via increased uptake of its valine ester prodrug through amino acid transporters. Xenobiotica 42:603-613.

Lin X, Skolnik S, Chen X, and Wang J (2011) Attenuation of intestinal absorption by major efflux transporters: quantitative tools and strategies using a Caco-2 model. Drug Metab Dispos 39: 265-274.

Livak KJ and Schmittgen TD (2001) Analysis of relative gene expression data using real-time quantitative PCR and the 2(-delta delta C(T)) method. Methods 25:402-408.

Lowry OH, Rosebrough NJ, Farr AL, and Randall RJ (1951) Protein measurement with the Folin phenol reagent. J Biol Chem 193:265-275.

Maliepaard M, Scheffer GL, Faneyte IF, van Gastelen MA, Pijnenborg AC, Schinkel AH, van De Vijver MJ, Scheper RJ, and Schellens JH (2001) Subcellular localization and distribution of the breast cancer resistance protein transporter in normal human tissues. Cancer Res 61:3458-3464.

Matsushima S, Maeda K, Kondo C, Hirano M, Sasaki M, Suzuki H, and Sugiyama Y (2005) Identification of the hepatic efflux transporters of organic anions using double-transfected Madin-Darby canine kidney II cells expressing human organic anion-transporting polypeptide 1B1 (OATP1B1)/multidrug resistance-associated protein 2, OATP1B1/multidrug resistance 1, and OATP1B1/breast cancer resistance protein. J Pharmacol Exp Ther 314:1059-1067.

Miller AD and Buttimore C (1986) Redesign of retrovirus packaging cell lines to avoid recombination leading to helper virus production. Mol Cell Biol 6:2895-2902.

Miyake K, Mickley L, Litman T, Zhan Z, Robey R, Cristensen B, Brangi M, Greenberger L, Dean M, Fojo T, et al. (1999) Molecular cloning of cDNAs which are highly overexpressed in mitoxantrone-resistant cells: demonstration of homology to $\mathrm{ABC}$ transport genes. Cancer Res 59:8-13.

Mohrmann K, van Eijndhoven MA, Schinkel AH, and Schellens JH (2005) Absence of N-linked glycosylation does not affect plasma membrane localization of breast cancer resistance protein (BCRP/ABCG2). Cancer Chemother Pharmacol 56:344-350.

Nakanishi T, Doyle LA, Hassel B, Wei Y, Bauer KS, Wu S, Pumplin DW, Fang HB, and Ross DD (2003a) Functional characterization of human breast cancer resistance protein (BCRP, ABCG2) expressed in the oocytes of Xenopus laevis. Mol Pharmacol 64:1452-1462.

Nakanishi T, Karp JE, Tan M, Doyle LA, Peters T, Yang W, Wei D, and Ross DD (2003b) Quantitative analysis of breast cancer resistance protein and cellular resistance to flavopiridol in acute leukemia patients. Clin Cancer Res 9:3320-3328.

Nakanishi T and Ross DD (2012) Breast cancer resistance protein (BCRP/ABCG2): its role in multidrug resistance and regulation of its gene expression. Chin J Cancer 31:73-99.
Nakatomi K, Yoshikawa M, Oka M, Ikegami Y, Hayasaka S, Sano K, Shiozawa K, Kawabata S, Soda H, Ishikawa T, et al. (2001) Transport of 7-ethyl-10-hydroxycamptothecin (SN-38) by breast cancer resistance protein ABCG2 in human lung cancer cells. Biochem Biophys Res Commun 288:827-832.

Natarajan K, Xie Y, Baer MR, and Ross DD (2012) Role of breast cancer resistance protein (BCRP/ABCG2) in cancer drug resistance. Biochem Pharmacol 83:1084-1103.

Niu N and Wang L (2015) In vitro human cell line models to predict clinical response to anticancer drugs. Pharmacogenomics 16:273-285.

Oguma T (2001) Antitumor drugs possessing topoisomerase I inhibition: applicable separation methods. J Chromatogr B Biomed Sci Appl 764:49-58.

Shoemaker RH (2006) The NCI60 human tumour cell line anticancer drug screen. Nat Rev Cancer 6:813-823.

Vichai V and Kirtikara K (2006) Sulforhodamine B colorimetric assay for cytotoxicity screening. Nat Protoc 1:1112-1116.

Volk EL, Rohde K, Rhee M, McGuire JJ, Doyle LA, Ross DD, and Schneider E (2000) Methotrexate cross-resistance in a mitoxantrone-selected multidrug-resistant MCF7 breast cancer cell line is attributable to enhanced energy-dependent drug efflux. Cancer Res 60:3514-3521.

Xiao Y, Davidson R, Smith A, Pereira D, Zhao S, Soglia J, Gebhard D, de Morais S, and Duignan DB (2006) A 96-well efflux assay to identify ABCG2 substrates using a stably transfected MDCK II cell line. Mol Pharm 3:45-54.

Zhang J, Zhang L, Yan Y, Li S, Xie L, Zhong W, Lv J, Zhang X, Bai Y, and Cheng Z (2015) Are capecitabine and the active metabolite 5-Fu CNS penetrable to treat breast cancer brain metastasis? Drug Metab Dispos 43:411-417.

Zhou L, Schmidt K, Nelson FR, Zelesky V, Troutman MD, and Feng B (2009) The effect of breast cancer resistance protein and P-glycoprotein on the brain penetration of flavopiridol, imatinib mesylate (Gleevec), prazosin, and 2-methoxy-3-(4-(2-(5-methyl-2-phenyloxazol-4-yl)ethoxy)phenyl)propanoic acid (PF-407288) in mice. Drug Metab Dispos 37:946-955.

Address correspondence to: Dr. Ikumi Tamai, Faculty of Pharmaceutical Sciences, Institute of Medical, Pharmaceutical and Health Sciences, Kanazawa University, Kakuma-machi, Kanazawa 920-1192, Japan. E-mail: tamai@p.kanazawa-u.ac.jp 